\title{
Cholesterol Nucleation Time in Gallbladder Bile of Patients with Solitary or Multiple Cholesterol Gallstones
}

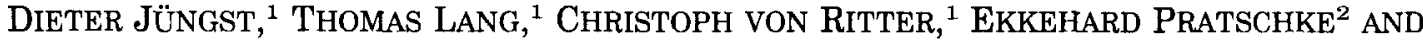 \\ GUSTAV PAUMGARTNER \\ Departments of ${ }^{2}$ Medicine II and ${ }^{2}$ Surgery, Klinikum Grosshadern, Ludwig-Maximilians-University, Munich, 8000 Munich \\ 70, Germany
}

\begin{abstract}
Patients with multiple cholesterol gallbladder stones have been found to be at a higher risk for the recurrence of gallstones after successful nonsurgical treatment than those with a solitary stone. Cholesterol gallstone recurrence, like primary gallstone formation, probably involves a triple defect with supersaturation, abnormally rapid nucleation of cholesterol in bile and altered gallbladder motor function. We investigated whether the increased recurrence rate of patients with multiple stones might be caused by more rapid nucleation. Therefore the time required for cholesterol monohydrate crystals to appear in ultracentrifuged bile of patients with solitary $(n=71)$ or multiple $(n=42)$ cholesterol gallstones was determined.
\end{abstract}

The cholesterol nucleation time was significantly $(p<0.01)$ longer in the bile from patients with solitary stones ( $<1$ to 16 days, median $=2.0$ days) than in the bile from patients with multiple stones $(<1$ to 8 days, median $=1.0$ days). Moreover, 15 of $71(21.1 \%)$ patients with solitary cholesterol stones but only 1 of $42(2.4 \%)$ patients with multiple cholesterol stones showed a normal ( $>4$ days) nucleation time.

However, no difference in the cholesterol saturation index was found between the bile samples from patients with solitary stones and the bile samples from patients with multiple stones $(1.55 \pm 0.65$ vs. $1.54 \pm$ 0.59 , mean \pm S.D., respectively). The more rapid cholesterol nucleation in gallbladder bile may, therefore, be the major risk factor causing the higher percentage of stone recurrence in patients with multiple cholesterol stones as compared with patients with solitary cholesterol stones. (HEPATOLOGY 1992;15:804-808.)

The recurrence of cholesterol gallstones, like primary gallbladder stone formation, probably involves a triple defect with cholesterol supersaturation, abnormally

Received October 22, 1990; accepted November 25, 1991.

Part of this work was presented at the 24th Annual Meeting of the European Association for the Study of Liver Diseases in Munich, Germany, August 30-September 2, 1989, and an abstract appeared in Journal of Hepatology 1989;9(suppl.1):S51.

Address reprint requests to: Dieter Jüngst, M.D., Department of Medicine II, Klinikum Grosshadern, Marchioninistr. 15, 8000 Munich 70, Germany. 31/1/35676 rapid nucleation and impaired gallbladder motor function (1-6).

Recurrence rates of $30 \%$ to $61 \%$ over 3 to $12 \mathrm{yr}$ have been reported after successful nonsurgical treatment of gallstones (7-10). The recurrence rate increases about $10 \%$ a year, and after 3 to $5 \mathrm{yr}$ a plateau with no further recurrences is usually seen $(7,8)$. Multiple gallstones seem to recur more often than solitary stones (9). The mechanism underlying this observation is unclear but might be related to different nucleation times in gallbladder bile. Therefore we measured cholesterol nucleation time in the gallbladder bile of patients with multiple or solitary cholesterol gallstones to test whether the increased risk of patients with multiple stones is caused by more rapid nucleation.

\section{MATERIALS AND METHODS}

Patients and Collection of Bile Samples. A total of 113 patients, 85 women and $28 \mathrm{men}$, who underwent elective cholecystectomy because of symptomatic gallstone disease were included in the study. A total of 71 patients (51 women and $20 \mathrm{men}$ ) had a solitary gallbladder stone with a cholesterol content above $50 \%$, and 42 patients ( 34 women and 8 men) had multiple stones with a cholesterol content above $50 \%$. Twelve patients with dilute gallbladder bile (total lipid concentration (TLC) $<1.0 \mathrm{gm} / \mathrm{dl}$ ) and suspected cystic duct obstruction were excluded from the study. During surgery bile was aspirated from the gallbladder with an 18-gauge needle soon after ligation of the cystic duct. Particular care was taken to collect gallbladder bile completely. Because of the known stratification of human gallbladder bile (11), only complete aspiration of bile can provide valid samples. The clamping of the gallbladder wall before bile aspiration was avoided to prevent mucosal damage. Stones were removed with the gallbladder, washed with distilled water, dried and weighed. The cholesterol content of the stones was measured chemically after extraction with organic solvents and was expressed as percentage of dry weight (12).

Cholesterol Nucleation Time. After collection bile samples were mixed thoroughly, and one drop was immediately examined by polarized light microscopy for cholesterol crystals.

For the determination of cholesterol nucleation time, $4 \mathrm{ml}$ of gallbladder bile was centrifuged at $37^{\circ} \mathrm{C}$ for $1 \mathrm{hr}$ at $100,000 \mathrm{~g}$ in a Beckman L-50 ultracentrifuge (Beckman Instruments, Fullerton, CA) to obtain crystal-free bile as described by Holan et al. (3). The top $1 \mathrm{ml}$ was discarded, and 
Table 1. Concentrations of bile acids, phospholipids, cholesterol, total lipids and the CSI in 17 gallbladder bile samples from patients with cholesterol gallstones after ultracentrifugation and after ultrafiltration (Millex 0.22- $\mu \mathrm{m}$ filter)

\begin{tabular}{lcc}
\hline & \multicolumn{2}{c}{ Gallbladder bile samples } \\
\cline { 2 - 3 } & $\begin{array}{c}\text { After } \\
\text { ultracentrifugation }\end{array}$ & $\begin{array}{c}\text { After } \\
\text { ultrafiltration }\end{array}$ \\
\hline Bile acids $(\mathrm{mmol} / \mathrm{L})$ & $111.9 \pm 61.4$ & $74.4 \pm 45.0^{a}$ \\
Phospholipids $(\mathrm{mmol} / \mathrm{L})$ & $38.2 \pm 23.6$ & $21.0 \pm 14.9^{a}$ \\
Cholesterol $(\mathrm{mmol} / \mathrm{L})$ & $16.9 \pm 9.4$ & $8.4 \pm 5.9^{a}$ \\
Total lipids $(\mathrm{gm} / \mathrm{dl})$ & $9.2 \pm \mathbf{5 . 2}$ & $5.4 \pm 3.6^{a}$ \\
CSI & $1.52 \pm 0.48$ & $1.32 \pm \mathbf{0 . 4 1}$ \\
\hline
\end{tabular}

All numbers mean \pm S.D.

${ }^{a} \mathrm{p}<0.001$.

the next $2 \mathrm{ml}$ (interphase) was removed by aspiration. Cultures of the native bile were examined for bacterial growth. In addition, $10 \mu \mathrm{l}$ of $20 \%$ sodium azide was added to $1.0 \mathrm{ml}$ bile aliquots (interphase) to prevent bacterial growth during incubation. An aliquot of the interphase was immediately examined microscopically to confirm the absence of crystals; the rest was placed in sterile tubes, flushed with nitrogen, sealed and incubated at $37^{\circ} \mathrm{C}$. The interphase was investigated daily for the appearance of cholesterol crystals.

The interval between time zero and the first detection of a cholesterol crystal in the sample was taken as the nucleation time. In accordance with Holan et al. (3) a discriminating point in time at 4 days was chosen to differentiate between normal $(>4$ days) and abnormal ( $\leq 4$ days) nucleation time of cholesterol in bile.

For the sake of comparison, additional determinations of cholesterol nucleation time were performed in 17 selected samples of gallbladder bile from patients with cholesterol stones using the method described by van Erpecum et al. (13). These authors used ultrafiltration with $0.22-\mu \mathrm{m}$ Millex filters (Millipore, Molsheim, France) instead of ultracentrifugation to remove cholesterol crystals from the bile samples. In the 17 patients nucleation times in the bile samples after ultrafiltration were about threefold to fourfold longer than in ultracentrifuged bile samples (Fig. 1). As shown in Table 1 a highly significant decrease of the main biliary lipids (bile acids, phospholipids and cholesterol) was found in ultrafiltered as compared with ultracentrifuged bile samples. These results demonstrate that ultrafiltration of gallbladder bile even through large pore-sized membranes may alter the composition of bile, leading to a prolongation of cholesterol nucleation time. We therefore used ultracentrifugation instead of ultrafiltration in our study to remove cholesterol crystals from the bile samples before the nucleation assay.

Analysis of Biliary Lipids. For the analysis of biliary lipids, duplicate aliquots were stored at $-70^{\circ} \mathrm{C}$ before determination. Cholesterol was determined colorimetrically with the Liebermann-Burchard reaction after the extraction of a 1-ml methanolic bile sample using petroleum ether (14). Phospholipids were measured as total biliary phosphate after hydrolysis at $150^{\circ} \mathrm{C}$ with sulfuric acid using the colorimetric assay of Fiske and Subbarow (15). Inorganic phosphate is only a trace constituent of total biliary phosphate; therefore total phosphate adequately reflects lipid-bound phosphate. Total bile salts were determined by a modified 3 - $\alpha$-hydroxysteroid dehydrogenase method (16).

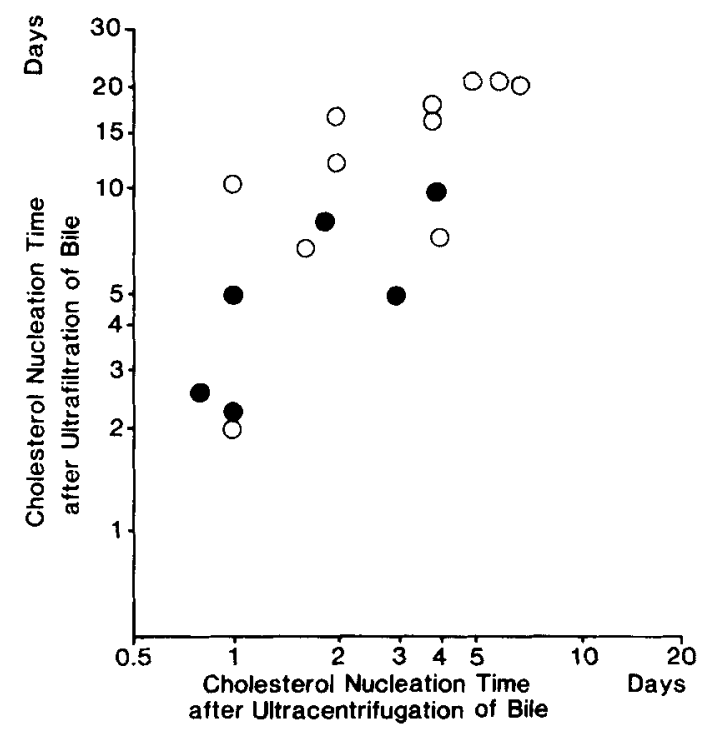

FIG. 1. Relation of cholesterol nucleation time (logarithmic scale) after ultracentrifugation and after ultrafiltration of gallbladder bile from 11 patients with solitary $(0)$ and 6 patients with multiple (•) cholesterol stones.

The cholesterol saturation index (CSI) of each sample was calculated by dividing the cholesterol concentration by the maximum cholesterol solubility according to Carey (17) and corrected for the total lipid content of each individual bile sample.

Statistical Analysis. Student's $t$ test was used for parametric data and the Mann-Whitney $U$ test was applied for nonparametric data (nucleation time). Nonparametric correlation was performed by Spearman's rank correlation coefficient. The level of statistical significance was set at $p \leq 0.05$.

\section{RESULTS}

The concentrations of bile acids, phospholipids, cholesterol, total lipids, CSI and the cholesterol nucleation time in the different groups of patients are shown in Table 2. In the bile of patients with solitary or multiple cholesterol gallstones, similar concentrations of the biliary lipids and the CSI values were determined. However, the cholesterol nucleation time was significantly $(p<0.01)$ longer in the bile samples of patients with solitary cholesterol gallstones than in the bile samples of patients with multiple cholesterol gallstones (Table 2). Only $1(2.4 \%)$ of 42 patients with multiple cholesterol gallstones but $15(21.1 \%)$ of 71 patients with solitary cholesterol gallstones showed a normal $(>4$ days) nucleation time (Fig. 2A, B) in the bile samples. No correlation was found between the cholesterol nucleation time and the CSI in both groups of patients (Fig. $2 \mathrm{~A}, \mathrm{~B})$. Indeed, in some bile samples the CSI demonstrated apparent undersaturation with cholesterol, yet an abnormally rapid nucleation of cholesterol was determined (Fig. 2A, B).

Dilute bile samples, as expected, showed higher CSI values than more concentrated bile samples, and a significant $(p<0.02)$ negative correlation between TLC and CSI was seen in gallbladder bile samples of both 

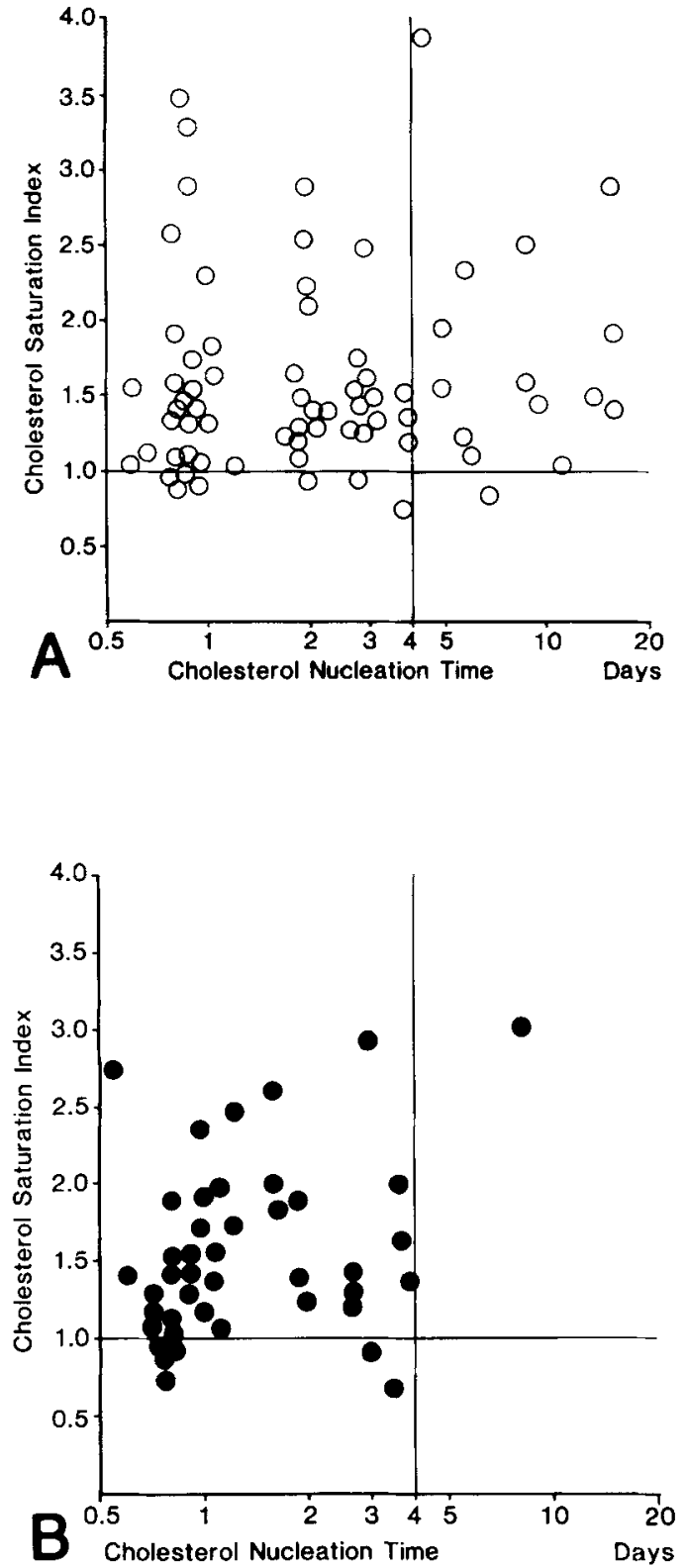

FIG. 2. Relation of cholesterol nucleation time (logarithmic scale) and CSI in the gallbladder bile samples from 71 patients with (A) solitary $(O)$ and 42 patients with (B) multiple ( $)$ cholesterol stones.

patients with solitary and multiple cholesterol stones (Fig. 3A, B). Despite this finding, the cholesterol nucleation times in dilute $(<5 \mathrm{gm} / \mathrm{dl}$ TLC) bile samples $(<1$ to 16 days, median $=2.0$ days) tended to be even longer than in more concentrated ( $>5 \mathrm{gm} / \mathrm{dl}$ TLC) bile samples ( $<1$ to 9 days, median $=1.5$ days $)$, but no correlation between the single values of TLC and cholesterol nucleation time in bile samples was obtained (data not shown).

\section{DISCUSSION}

In a recent study by Villanova et al. (9) solitary gallbladder stones were found to have less tendency to
TABLe 2. Concentration of bile acids, phospholipids, cholesterol, total lipids and CSI and cholesterol nucleation time in gallbladder bile samples from 71 patients with solitary cholesterol gallstones and 42 patients with multiple cholesterol gallstones

\begin{tabular}{lcc}
\hline & \multicolumn{2}{c}{ Gallbladder bile samples } \\
\cline { 2 - 3 } & $\begin{array}{c}\text { Patients } \\
\text { with solitary } \\
\text { cholesterol stones } \\
(\mathbf{n}=\mathbf{7 1})\end{array}$ & $\begin{array}{c}\text { Patients } \\
\text { with multiple } \\
\text { cholesterol stones } \\
(\mathbf{n}=\mathbf{4 2})\end{array}$ \\
\hline Bile acids (mmol/L) & $79.2 \pm \mathbf{4 2 . 1}$ & $75.1 \pm 46.3$ \\
Phospholipids (mmol/L) & $29.6 \pm 18.4$ & $28.1 \pm 18.9$ \\
Cholesterol (mmol/L) & $12.2 \pm 5.9$ & $11.4 \pm 7.6$ \\
Total lipids (gm/dl) & $6.7 \pm 3.4$ & $6.4 \pm 3.8$ \\
CSI & $1.55 \pm 0.65$ & $1.54 \pm 0.59$ \\
Nucleation time (days) & 2.0 (median) $^{a}$ & 1.0 (median) $^{b}$ \\
\hline
\end{tabular}

All numbers mean \pm S.D.

${ }^{a}$ Range $=$ less than 1 day up to 16 days.

${ }^{b}$ Range $=$ less than 1 day up to 8 days; $\mathrm{p}<0.01$.

recur after successful medical treatment than multiple stones. In an attempt to explain the pathogenetic factors responsible for the higher recurrence rate of multiple gallbladder stones, we compared cholesterol saturation and the cholesterol nucleation time in bile samples of 71 patients with solitary gallstones and 42 patients with multiple gallstones. No differences in cholesterol saturation were found between bile samples from patients with solitary stones and bile samples from patients with multiple stones. Nucleation time, however, was significantly shorter in bile samples with multiple cholesterol stones than in bile samples with solitary gallbladder stones. Only 1 of 42 patients with multiple stones as compared with 15 of 71 patients with a single stone had a normal nucleation time ( $>4$ days). On the other hand, a rapid nucleation time of 1 day and less was observed in only $35 \%$ of patients with solitary cholesterol stones compared with $50 \%$ of patients with multiple cholesterol stones. These rates are very similar to the percentage of long-term stone recurrence after successful dissolution with oral bile acids. These observations suggest that an abnormally rapid nucleation of cholesterol in gallbladder bile is associated with a high rate of stone recurrence.

Although our results confirm in a larger group of patients earlier observations by Gollish et al. (18), our findings show marked differences compared with a study by van Erpecum et al. (13). The latter group observed longer nucleation times both in a group of 45 patients with multiple cholesterol stones ( 1 to more than 21 days; median $=4.0$ days) and in 22 patients with solitary cholesterol stones ( 2 to more than 21 days; median $=21$ days). This clear discrepancy may be the result of the difference in methods used in performing the cholesterol nucleation assay. In place of ultracentrifugation as originally described by Holan et al. (3) and as used by Gollish et al. (18) and ourselves, van Erpecum et al. (13) used ultrafiltration with $0.22-\mu \mathrm{m}$ Millex filters to remove cholesterol crystals from bile. As shown in Table 1 we found a significant decrease in all types of biliary 
lipids after ultrafiltration (bile acids, phospholipids and cholesterol), which led to a slight decline in the CSI and obviously to a prolonged nucleation time in ultrafiltered as compared with ultracentrifuged bile samples. Conceivably, the used filters retain cholesterol-phospholipid vesicles and mixed micellar lipid aggregates.

The retention of bile lipids by a $0.22-\mu \mathrm{m}$ filter has not been reported by Pattinson and Willis (19) and Whiting and Watts (20). Pattinson and Willis (19) compared filtered and ultracentrifuged bile in only 4 of a total of 11 bile samples because, as a result of high mucus content, ultrafiltration did not yield sufficient bile in 7 samples. The data on 4 of the 11 samples may therefore not be representative. Nevertheless, Pattinson and Willis (19) confirmed that the cholesterol nucleation time in ultrafiltered bile is consistently prolonged compared with ultracentrifuged bile $(9.3 \pm 3.7$ days vs. $2.9 \pm 0.4$ days $)$ as has been shown in our study. In the study by Whiting and Watts (20) no data are given.

Groen et al. (21), using concanavalin A-Sepharose chromatography of gallbladder bile, isolated a glucosecontaining and mannose-containing $130-\mathrm{kD}$ glycoprotein with strong cholesterol nucleation-promoting activity in model bile samples. This glycoprotein was found in most gallbladder bile samples, but much higher nucleation-promoting activity titers were observed in bile samples from patients with multiple cholesterol stones than in bile samples from patients with solitary stones. It is tempting to speculate that this observation could partially explain the differences in cholesterol nucleation time in the gallbladder bile samples of patients with solitary gallstones and multiple gallstones found in our study.

Cholesterol supersaturation of bile is thought to be the prerequisite for gallstone formation $(1,2)$. However, in our study some patients with apparent cholesterol undersaturation showed clearly abnormal nucleation times (Fig. 3A, B). This observation is in accordance with two earlier studies showing cholesterol crystal growth in gallbladder bile samples with a CSI below 1.0. In a group of five patients with a CSI between 0.8 and 0.9 , Schriever and Jüngst (22) observed cholesterol monohydrate crystals in gallbladder bile. Similar data were collected by Whiting and Watts (20), who determined the cholesterol solubility limit of human gallbladder bile by seeding bile samples with a small amount of cholesterol crystals. The reason for those findings is unclear but again demonstrates the limitations of the CSI in predicting cholesterol solubility in native bile.

In accordance with our data van Erpecum et al. (13) found that bile concentration is inversely correlated with the CSI. A recent study by Shiffman, Sugerman and Moore (23) confirms this observation. In that study the CSI in gallbladder bile samples of 78 morbidly obese patients decreased curvilinearly from a maximum of 3.7 in dilute bile to 1.0 to 1.5 in concentrated bile. In our study we did not find a significant effect of TLCs on cholesterol nucleation time. This is in accordance with the in vivo and in vitro results of Gollish et al. (18) but contrary to the observations of van Erpecum et al. (13).
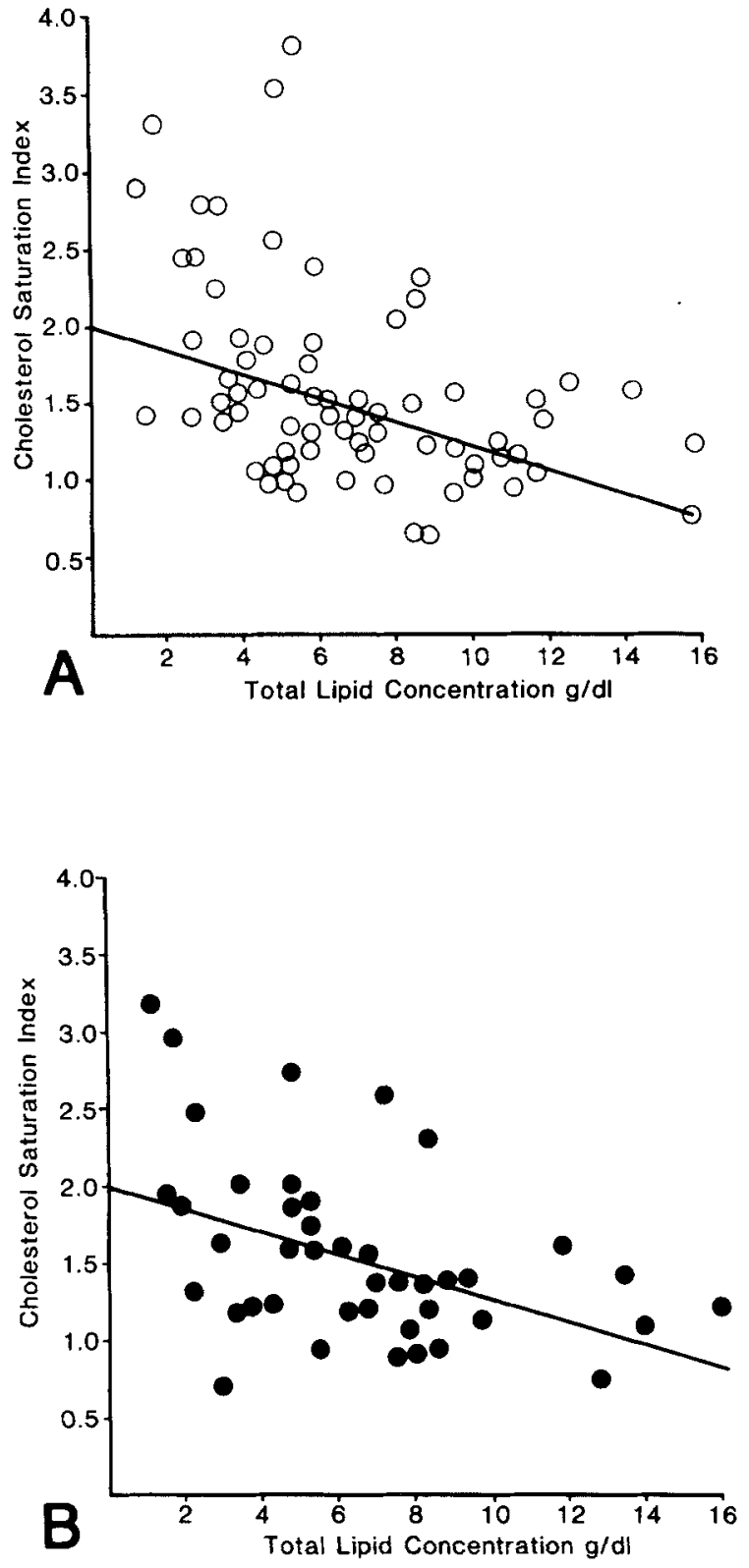

Fig. 3. Relation of TLC and CSI in gallbladder bile samples from 71 patients with (A) solitary $(0)(r=-0.38 ; p<0.02)$ and 42 patients with (B) multiple (*) cholesterol stones $(\mathrm{r}=-0.44 ; \mathrm{p}<0.02)$.

The use of ultrafiltration rather than ultracentrifugation in the study of van Erpecum et al. (13) before the nucleation assay might be responsible for these discrepancies.

In conclusion, our data show a similar composition and concentration of biliary lipids in bile samples from patients with solitary vs. multiple cholesterol gallstones but a significant difference in the nucleation time. The rates of rapid nucleation ( $\leq 1$ day) are similar to the percentage of long-term stone recurrence and suggest that rapid nucleation of cholesterol in gallbladder bile might be a major risk factor for recurrent cholesterol stones. 
Acknowledgments: We thank Benedikta Zündt for her excellent technical assistance and her help in the preparation of the manuscript and Florian Ringel for statistical analysis.

\section{REFERENCES}

1. Admirand WH, Small DM. The physicochemical basis of cholesterol gallstone formation in man. J Clin Invest 1968;47:10431052.

2. Carey MC, Small DM. The physical chemistry of cholesterol solubility in bile. J Clin Invest 1978;61:998-1026.

3. Holan KR, Holzbach RT, Hermann RE, Cooperman AM, Claffey WY. Nucleation time: a key factor in the pathogenesis of cholesterol gallstone disease. Gastroenterology 1979;77:611617.

4. Sedaghat A, Grundy SM. Cholesterol crystals and the formation of cholesterol gallstones. N Engl J Med 1980;302:1274-1277.

5. Pomeranz I, Shaffer E. Abnormal gallbladder emptying in a subgroup of patients with gallstones. Gastroenterology 1985;88: $787-791$.

6. Spengler U, Sackmann M, Sauerbruch T, Holl J, Paumgartner G. Gallbladder motility before and after extracorporeal shock-wave lithotripsy. Gastroenterology 1989;96:860-863.

7. Lanzini A, Jazrawi RP, Kupfer RM, Mangdal DP, Joseph AEA, Northfield TC. Gallstone recurrence after medical dissolution: an overestimated threat? J Hepatol 1986;3:241-246.

8. O'Donnel LDJ, Heaton KW. Recurrence and re-recurrence of gallstones after medical dissolution: a longterm follow up. Gut 1988;29:655-658.

9. Villanova N, Bazzoli F, Taroni F, Frabboni R, Mazzella G, Festi D, Barbara L, et al. Gallstone recurrence after successful oral bile acid treatment: a 12-year follow-up study and evaluation of long-term postdissolution treatment. Gastroenterology 1989;97: 726-731.

10. Sackmann M, Ippisch E, Sauerbruch T, Holl J, Brendel W, Paumgartner G. Early gallstone recurrence rate after successful shock-wave therapy. Gastroenterology 1990;98:392-396.
11. Tera H. Stratification of human gallbladder bile in vivo. Acta Chir Scand 1960;256(suppl.):4-85.

12. Smallwood RA, Jablonshi $P$, Watts JM. Intermittent secretion of abnormal bile in patients with cholesterol gallstones. BMJ 1972;4:263-266.

13. Van Erpecum KJ, van Berge Henegouwen GP, Stoelwinder B, Schmidt YMG, Willekens FLH. Bile concentration is a key factor for nucleation of cholesterol crystals and cholesterol saturation index in gallbladder bile of gallstone patients. HEPATOLOGY 1990;11:1-6.

14. Abell LL, Levy BB, Brodie BB, Kendall FE. A simplified method for the estimation of total cholesterol in serum and demonstration of its specificity. J Biol Chem 1952;195:357-366.

15. Fiske $\mathrm{CH}$, Subbarow $\mathrm{Y}$. The colorimetric determination of phosphorus. J Biol Chem 1925;66:375-400.

16. Talalay P. Enzymatic analysis of steroid hormones. Methods Biochem Anal 1960;8:119-143.

17. Carey MC. Critical tables for calculating the cholesterol saturation of native bile. J Lipid Res 1978;19:945-955.

18. Gollish SH, Burnstein MJ, Ilson RG, Petrunka CN, Strasberg SM. Nucleation of cholesterol monohydrate crystals from hepatic and gallbladder bile of patients with gallstones. Gut 1983;24:836-844.

19. Pattinson NR, Willis KE. Nucleation of cholesterol crystals from native bile and the effect of protein hydrolysis. J Lipid Res 1991;32:215-221.

20. Whiting MJ, Watts JM. Supersaturated bile from obese patients without gallstones supports cholesterol crystal growth but not nucleation. Gastroenterology 1984;86:243-248.

21. Groen AK, Noordam C, Drapers JAG, Egbers P, Jansen PCM, Tytgat GNJ. Isolation of a potent cholesterol nucleationpromoting activity from human gallbladder bile: role in the pathogenesis of gallstone disease. HePATOLOGY 1990;11:525-533.

22. Schriever CE, Jüngst $D$. Association between cholesterolphospholipid vesicles and cholesterol crystals in human gallbladder bile. HEPATOLOGY 1989;9:541-546.

23. Shiffman ML, Sugerman HJ, Moore EW. Human gallbladder mucosal function: effect of concentration and acidification of bile on cholesterol and calcium solubility. Gastroenterology 1990;99: 1452-1459. 